\title{
Acute exacerbation in COPD: we must do more
}

\author{
Tobias Welte
}

Affiliation: Dept of Respiratory Medicine and Member of the German Center for Lung Research (DZL), Medizinische Hochschule Hannover, Hannover, Germany.

Correspondence: Tobias Welte, Dept of Respiratory Medicine and Member of the German Center for Lung Research (DZL), Medizinische Hochschule Hannover, Carl- Neuberg-Strasse 1, 30625 Hannover, Germany.

E-mail: welte.tobiasamh-hannover.de

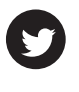

@ERSpublications

COPD audit: COPD exacerbation and pneumonia are internal medicine emergencies needing treatment in specialist units http://ow.ly/VgjSS

If one asks medical students which emergencies they hold as especially important in internal medicine, more than $90 \%$ first mention myocardial infarction and stroke. This is not surprising, because these diseases occupy the first places in the mortality statistics contained in the Global Burden of Disease report [1]. Nevertheless, respiratory failure is only described as rare, despite the fact that chronic obstructive pulmonary disease (COPD) is ranked fifth and pneumonia seventh in the same report. While the high hospital mortality rate of myocardial infarction and stroke is well known, the risk of acute exacerbation of COPD and of pneumonia is often underestimated.

Nationwide outcome registries for hospital mortality in myocardial infarction exist in Sweden and in the UK, demonstrating an in-hospital mortality of $7.6 \%$ and $10.5 \%$ [2]. For stroke it is higher, for several reasons; recently published data from the North Dublin population stroke study reported 20.7\% [3]. Data regarding mortality for hospital-admitted community acquired pneumonia vary widely, as a function of different methodologies used in different studies; on average it might be between 10 and 15\% [4]. Also for patients hospitalised with an acute exacerbation of COPD, numbers vary widely; a recently published systemic review reported mortality in the range between 1.8 and $20.4 \%$ with a mean of $3.6 \%$ [5]. In this issue of the European Respiratoy Journal, the first data from the European COPD audit are presented. This study included 16016 patients hospitalised with acute exacerbation of COPD from 422 hospitals in 13 European countries [6]. Mortality, at about 5\%, was higher than in most other studies, which may be explained by the high number of patients requiring mechanical ventilation (mostly noninvasive positive pressure ventilation), making it more likely that these patients had been more severely ill than those in other studies. This is in line with a recently published US study including only patients with acute exacerbation of COPD on mechanical ventilation, which showed a hospital mortality rate of 5.9\% [7].

Hospital mortality rates in patients suffering from myocardial infarction and stroke have decreased consistently over the past decades. One major reason for that was the foundation of coronary care and stroke units led by qualified staff and doctors, and offering standardised treatment approaches. For both units a dramatic improvement with regard to a number of outcome measures could be shown $[8,9]$. Meanwhile, this type of unity has been established nationwide in nearly all devolped countries. For respiratory failure, however, especially for patients with pneumonia and acute exacerbation of COPD who are not primarily admitted directly to an intensive care unit, nothing comparable has been set up. KolDitz et al. [10] analysed data from the German competence network for community acquired pneumonia (CAPNETZ) and found that mortality was up to $20 \%$ in patients with respiratory failure and increased further if the deterioration of the respiratory function starts later than 72 hours after hospital admission: demonstrating that treatment failure had been overlooked due to a lack of a standardised 
management plan for this patients. The COPD audit found that only $45.0 \%$ of the patients with mild and $77.2 \%$ with severe respiratory acidosis received mechanical ventilation, although the positive effect of this measure has been clearly demonstrated [11]. In addition, only $81.6 \%$ of patients with acute exacerbation of COPD underwent blood gas diagnostics at admission, although this has been strongly recommended in various guidelines. If it was not clear enough before, the COPD audit has finally demonstrated that the management of acute exacerbation of COPD is far away from being good in European hospitals.

One of the most important limitations in the management of acute exacerbation of COPD is the lack of a standardised operating procedure for these patients. While this is available on coronary care or stroke units on the basis of a huge number of randomised controlled trials, the evidence in diagnostics and treatment of acute exacerbation of COPD is low. Most recommendations, with the exception of those for noninvasive ventilation, relating to acute exacerbation of COPD in the American Thoracic Society/ European Respiratory Society guidelines [11] remain vague and are not based on reliable study data. A lot of questions remain unanswered. Should bronchodilators be used during the acute phase of acute exacerbation of COPD and, if yes, which ones and how should they delivered? Does a short term course of oral corticosteroids make sense for all patients with acute exacerbation of COPD and what should be done if oral treatment is not feasible? Who requires antibiotic therapy? Is there a place for antivirals? The most important problem, however, is the fact that most of the patients with acute exacerbation of COPD suffer from comorbidities, which often worsen during the acute exacerbation course [12]. The high readmission rate in the COPD audit is partly related to an inappropriate treatment of mainly cardiac comorbidities. More studies are necessary to develop a standardised diagnostic and therapeutic approach for the management of patients with acute exacerbation of COPD.

The COPD audit was necessary to show the deficits of care in patients with respiratory failure due to acute exacerbation of COPD. Audits can generate awareness of those problems and, consequently, improve quality of care. Therefore, audits should be made available for more hospitals. However, audits will not solve the problem of inadequate care of respiratory failure patients. This requires an understanding, from physicians, and also from healthcare politicians, that COPD exacerbation and pneumonia are internal medicine emergencies that need to be treated in specialist units.

\section{References}

1 Murray CJ, Vos T, Lozano R, et al. Disability-adjusted life years (DALYs) for 291 diseases and injuries in 21 regions, 1990-2010: a systematic analysis for the Global Burden of Disease Study 2010. Lancet 2012; 380: 2197-2223.

2 Chung SC, Gedeborg R, Nicholas O, et al. Acute myocardial infarction: a comparison of short-term survival in national outcome registries in Sweden and the UK. Lancet 2014; 383: 1305-1312.

3 Callaly E, Ni Chroinin D, Hannon N, et al. Rates, predictors, and outcomes of early and late recurrence after stroke: the North Dublin population stroke study. Stroke 2015; in press [DOI: STROKEAHA.115.011248].

4 Welte T, Torres A, Nathwani D. Clinical and economic burden of community-acquired pneumonia among adults in Europe. Thorax 2012; 67: 71-79.

5 Singanayagam A, Schembri S, Chalmers JD. Predictors of mortality in hospitalized adults with acute exacerbation of chronic obstructive pulmonary disease. Ann Am Thorac Soc 2013; 10: 81-89.

6 Hartl S, Lopez-Campos JL, Pozo-Rodriguez F, et al. Risk of death and readmission of hospital-admitted COPD exacerbations: European COPD Audit. Eur Respir J 2016; 47: 113-121.

7 Lindenauer PK, Stefan MS, Shieh MS, et al. Outcomes associated with invasive and noninvasive ventilation among patients hospitalized with exacerbations of chronic obstructive pulmonary disease. JAMA Intern Med 2014; 174: 1982-1993.

8 Morrow DA, Fang JC, Fintel DJ, et al. Evolution of critical care cardiology: transformation of the cardiovascular intensive care unit and the emerging need for new medical staffing and training models: a scientific statement from the American Heart Association. Circulation 2012; 126: 1408-1428.

9 Seenan P, Long M, Langhorne P. Stroke units in their natural habitat: systematic review of observational studies. Stroke 2007; 38: 1886-1892.

10 Kolditz M, Ewig S, Klapdor B, et al. Community-acquired pneumonia as medical emergency: predictors of early deterioration. Thorax 2015; 70: 551-558.

11 Celli BR, Decramer M, Wedzicha JA, et al. An official American Thoracic Society/European Respiratory Society statement: research questions in COPD. Eur Respir J 2015; 45: 879-905.

12 Papaioannou AI, Bartziokas K, Loukides S, et al. Cardiovascular comorbidities in hospitalised COPD patients: a determinant of future risk? Eur Respir J 2015; 46: 846-849. 